\title{
Typing TREX1 gene in patients with systemic lupus erythematosus
}

\author{
M. Fredii-3, M. Bianchi ${ }^{4}$, L. Andreoli ${ }^{1,2}$, G. Greco ${ }^{4}$, I. Olivieri' \\ S. Orcesi ${ }^{5}$, E. Fazzi ${ }^{6}$, C. Cereda ${ }^{4}$, A.Tincani $^{1,2}$ \\ ${ }^{1} R$ heumatology and Clinical Immunology Unit, Spedali Civili, Brescia; \\ 2Department of Clinical and Experimental Sciences, University of Brescia; \\ ${ }^{3}$ Rheumatology Chair, University of Pavia; \\ ${ }^{4}$ Laboratory of Experimental Neurobiology, C. Mondino National Institute of Neurology Foundation, Pavia; \\ ${ }^{5}$ Child Neurology and Psychiatry Unit, C. Mondino National Institute of Neurology Foundation, Pavia; \\ ${ }^{6}$ Child Neurology and Psychiatry Unit, Mother-Child Department, Civil Hospital, University of Brescia, Italy
}

\begin{abstract}
SUMMARY
An impaired expression of interferon- $\alpha$ regulated genes has been reported in patients with either systemic lupus erythematosus (SLE) or Aicardi-Goutières syndrome (AGS), a rare monogenic encephalopathy with onset in infancy. One of mutations causing AGS is located in the TREX1 gene on chromosome 3. Heterozygous mutations in TREXI were reported in SLE patients. TREXI is a DNA exonuclease with specificity for ssDNA. An impairment of its activity may result in the accumulation of nucleid acid. A recent study described a significant association between a haplotype including several common single nucleotide polymorphisms (SNPs) of TREXI and neurological manifestations in European SLE patients.

Fifty-one SLE patients were screened for TREX1 gene, and the corresponding data were collected from clinical charts.

A novel heterozygous variant (p.Asp130Asn) was identified in one patient and in none of 150 controls. A missense variation was located in one of the three active sites of the gene and was classified as probably damaging. Variations of SNP rs 11797 were detected in 33 SLE patients and a variation of rs 3135944 in one. A significantly higher rate of the minor allele (T nucleotide) of SNP rs11797 was found in SLE patients with neuropsychiatric manifestations [ $12 / 16(75 \%)$ vs 28/86 (32.5\%) $\mathrm{O}=0.002$, odds ratio $=6.4295 \%$ confidence interval $(1.7-26.2)]$. Only 1 out of 8 patients (12.5\%) with neuropsychiatric SLE carried the wild-type form in homozygosity. Although we analyzed a small number of patients, we found a novel variation of TREX1, which may be pathogenic. The polymorphism of rs 11797 was more frequent in SLE patients with neurological manifestations.
\end{abstract}

Key words: TREXI, Novel variation, Neuropsychiatric systemic lupus erythematosus.

Reumatismo, 2015; 67 (1): 1-7

\section{INTRODUCTION}

$T$ REXI (3' repair exonuclease 1) is the most abundant intracellular 3'-5'exonuclease in mammalian cells (1). It is located on chromosome $3 \mathrm{p} 21.31$ and consists of a single exon encoding for a 314-amino acid polypeptide. DNA exonuclease proofreads DNA polymerase and potentially also functions as a DNA-degrading enzyme in granzyme-A-mediated apoptosis (2). Furthermore, TREXI is involved in the restriction of endogenous retroviruses. $\mathrm{Mu}-$ tations of this enzyme could impair DNA damage repair, leading to the accumulation of endogenous retroelement-derived DNA
(3). A defective clearance of this DNA could induce interferon (IFN) production and an immune-mediated inflammatory response, promoting systemic autoimmunity. In fact, TREX1-deficient mice develop an inflammatory myocarditis and die of circulatory failure (4). In humans, mutations of the TREX1 gene have been identified in patients with Aicardi-Goutierès syndrome (AGS) $(5,6)$ and also in several autoimmune diseases, namely familial chilblain lupus (7), systemic lupus erythematosus (SLE) (8，9), Sjögren's syndrome and systemic sclerosis (10). AGS is a rare encephalopathy arising during the first year of life after an uneventful pregnancy (11).
Corresponding author: Micaela Fredi

P.le Spedali Civili, 1 25123 Brescia, Italy E-mail: elmic83@libero.it 
It is characterized by cerebral atrophy, acquired microcephaly, intracranial calcifications, leucodistrophy, lymphocytosis and raised level of INF- $\alpha$ in the cerebrospinal fluid. AGS is a genetic disease due to mutations in several genes, which are present in $90 \%$ of cases (12). Seven of these genes have been identified to date, namely: TREX1, RNASEH2A, RNASEH2B, RNASEH2C (which together encode for the ribonuclease $\mathrm{H} 2$ enzyme complex), SAMHDI, ADARI and IFIHI (coding for MDA5). These mutations include null alleles, frameshift mutations and non-synonymous changes in the catalytic domains and the C-terminal region. In AGS, most TREX1 mutations are autosomal recessive and diminish the exonuclease activity of enzymes (9). Patients with AGS can have autoimmune-like manifestations, such as lupus-like rash, chilblain lesions, arthritis, oral ulcers, thrombocytopenia, leukocytopenia and positive antinuclear antibodies that are frequently observed (13). For some patients with AGS a formal diagnosis of SLE according to the American College of Rheumatology (ACR) criteria for SLE was possible (14).

In SLE patients, most of the reported mutations of the TREXI gene are in heterozygosity and are usually located outside the catalytic domain in the C-terminal region. Previous studies have also analyzed the potential association of TREXI mutations and neuropsychiatric SLE (NPSLE). A relatively common risk haplotype of TREXI was described in European SLE patients with seizures (9) and a missense variant was identified in a patient with NPSLE (15). A number of data highlight the relationship between SLE and AGS. As a consequence, we decided to genotype the TREX1 gene in a cohort of SLE patients and evaluate possible associations between gene mutations and clinical and/or serological features.

\section{MATERIALS AND METHODS}

\section{Patients}

Fifty-one consecutive SLE patients attending the Rheumatology Unit of Bres- cia Hospital between 2011 and 2012 were enrolled in this study. The diagnoses were confirmed on the basis of the ACR criteria revised in 1997 (16) and the more recent Systemic Lupus International Collaborating Clinics classification (SLICC) criteria (17). Clinical and serological data were collected from clinical charts. The occurrence of clinical features was considered at any time during the follow-up. Data recorded in clinical charts were collected and classified in a database. Furthermore, we analyzed 150 healthy control subjects. This study was approved by the local Ethics Review Board.

\section{Mutation analysis}

Genomic DNA from patients and controls was extracted from peripheral blood using automatic standard procedures (Maxwell ${ }^{\circledR}$ 16 Blood DNA - Promega, Milan, Italy). TREX1 coding exon was amplified using primers located in adjacent intronic regions from genomic DNA by polymerase chain reaction (PCR). Primer sequences and PCR conditions are available upon request.

All amplicons were screened by direct sequencing using Big-Dye Terminator v3.1 sequencing kit (Applied Biosystems, Milan, Italy) and ABI 3130 Genetic Analyzer (Applied Biosystems). Each fragment was sequenced on both strands. The alignment to the reference sequence (NG_009820.1 RefSeqGene) was performed using Sequencher 4.8 software.

All the identified sequence variations were confirmed by sequencing forward and reverse independent PCR products. The presence of missense, synonymous and intronic variants was verified on the National Center for Biotechnology Information (NCBI) website and compared to literature data.

\section{Bioinformatics analysis and modelling}

Amplicon sequences were compared with the reference sequences in the NCBI database (http://www.ncbi.nlm.nih.gov). The TREX1 database can also be found under Leiden Open Variation Database. The effect of mutations on the protein structure or function was analyzed with the prediction program PolyPhen (http://genetics. 
bwh.harvard.edu/pph/) (18) and Project Hope software (http://www.cmbi.ru.nl/ hope) (19).

\section{Statistical analysis}

The two-tailed Student's $t$-test for continuous variables and Fisher's exact test or Yates's $\chi^{2}$ tests for categorical variables were used. $\mathrm{P}<0.05$ was considered statistically significant, and correction for multiple testing was carried out using the Bonferroni adjustment when required. The odds ratio and the $95 \%$ confidence interval (CI 95\%) were calculated.

\section{RESULTS}

All patients of the analyzed cohort (51 SLE cases) were females; the patients were unrelated and of Caucasian origin except one Chinese. All the controls were Caucasians. In our cohort, we detected variants of the TREX1 gene in 33 out of $51(64.7 \%)$ patients. In particular we reported the presence of two synonymous variants: rs3135944 (NM_033629.4:c.462T>C, NP_338599.1: p.Asp154Asp)andrs11797(NM_033629.4: c.531C >T, NP_338599.1:p.Tyr177Tyr), plus 1 non synonymous novel variant NM_033629.4:c.588G >A (NP 338599.1 :p.Asp130Asn).

The heterozygous missense variant was found in an anti-dsDNA and anti-Ro/SSA positive female SLE patient (1.96\%), with an age at disease onset of 25 years, followed in our Unit for 22 years with renal, hematological, thrombotic involvement. The mutation of GAC to AAC determined a substitution of aspartic acid with asparagine in the protein at position 130 (NP_338599.1:p.Asp130Asn). This residue is located in the highly conserved second exonuclease domain (Exo2), one of the three regions which form the catalytic site of the protein. This variation, which was never described before, was not found in any of the 150 Italian healthy subjects.

We used two different software applications to predict the potential functional role of the novel variation. Based on Polyphen scores, this mutation is probably damaging the protein (Hum Var 1/1 and Hum Div 1/1). This de novo mutation introduces an asparagine residue, which causes a charge variation.

The wild-type residue is negatively charged, whereas the mutant residue is neutral. Because the negative charge is lost, the interaction with the metal will be less stable, disturbing the domain.

The other two detected variations were synonymous. The rs11797 polymorphism is a common single nucleotide polymorphism (SNP), found in our cohort in 33 out 51 patients $(64.7 \%)$, with a minor allele frequency of $39.2 \%$ in the patients and $39.6 \%$ in the controls. We reported both homozygous and heterozygous mutations of this SNP, with similar rates in patients and controls. The other SNP rs3135944 is a rare variant, and in our cohort was found in heterozygosity in one patient also carrying the rs11797 variation.

We then looked for clinical and serological differences among patients with the wildtype versus the mutated form of the gene (Tab. I), evaluating the difference in the rate of the minor allele. This analysis was possible only for the rs11797 SNP, due to the low prevalence of the other two mutations in our cohort.

As shown in Table II, we reported a significantly higher rate of the minor allele (T nucleotide) of SNP rs11797 in SLE patients with neuropsychiatric manifestations compared to those without. Moreover, the assessment of the genotype distribution in

Table I - TREX1 variants observed in our cohort and their genotype frequency among patients and controls.

\begin{tabular}{|l|l|l|l|l|l|l|l|}
\hline $\begin{array}{l}\text { Nucleotide } \\
\text { variation }\end{array}$ & $\begin{array}{l}\text { Amino acid } \\
\text { change }\end{array}$ & $\begin{array}{l}\text { SLE } \\
\text { HZ wild type }\end{array}$ & $\begin{array}{l}\text { SLE } \\
\text { HTZ }\end{array}$ & $\begin{array}{l}\text { SLE } \\
\text { HZ mutated }\end{array}$ & $\begin{array}{l}\text { Control } \\
\text { HZ wild type }\end{array}$ & $\begin{array}{l}\text { Control } \\
\text { HTZ }\end{array}$ & $\begin{array}{l}\text { Control } \\
\text { HZ mutated }\end{array}$ \\
\hline c.462T>C & pAsp154Asp & $50 / 51(98 \%)$ & $1 / 51(2 \%)$ & $0 / 51(0 \%)$ & $150 / 150(100 \%)$ & $0 / 150(0 \%)$ & $0 / 150(0 \%)$ \\
\hline c.531C>T & p.Tyr177Tyr & $18 / 51(35.3 \%)$ & $26 / 51(51 \%)$ & $7 / 51(13.5 \%)$ & $50 / 150(33 \%)$ & $81 / 150(54 \%)$ & $19 / 150(12.6 \%)$ \\
\hline g.6214G $>$ A & p.Asp130Asn & $0 / 51(0 \%)$ & $1 / 51(2 \%)$ & $0 / 51(0 \%)$ & $0 / 150(0 \%)$ & $0 / 150(0 \%)$ & $0 / 150(0 \%)$ \\
\hline
\end{tabular}

SLE, systemic lupus erythematosus; HZ, homozygous; HTZ, heterozygous. 
Table II - Clinical features of our patients. We reported the frequency of the minor allele and the genotype distribution of the rs 11797 SNP for each feature.

\begin{tabular}{|c|c|c|c|c|c|c|}
\hline $\begin{array}{l}\text { Manifestation } \\
\text { (n. pts) }\end{array}$ & \begin{tabular}{|l} 
MAF (T) \\
frequency in \\
patient without \\
the manifestation
\end{tabular} & \begin{tabular}{|l|} 
MAF $(T)$ \\
frequency in \\
patients with the \\
manifestation
\end{tabular} & \begin{tabular}{|l} 
CC genotype \\
frequency in \\
patients with the \\
manifestation
\end{tabular} & \begin{tabular}{|l|} 
CT genotype \\
frequency in \\
patients with the \\
manifestation
\end{tabular} & $\begin{array}{l}\text { TT genotype } \\
\text { frequency in } \\
\text { patients with the } \\
\text { manifestation }\end{array}$ & $\begin{array}{l}\text { P value* } \\
\text { (comparison } \\
\text { MAF patient } \\
\text { with vs without } \\
\text { manifestation) }\end{array}$ \\
\hline $\begin{array}{l}\text { Artralgia/arthritis } \\
(45 / 51)\end{array}$ & $5 / 12(41.6)$ & $35 / 90(38.8)$ & $16 / 45(35.5)$ & $23 / 45(51.1)$ & $6 / 45(13.4)$ & ns \\
\hline Malar rash (19/51) & 22/64 (34.3) & $18 / 38(50)$ & $3 / 19(15.8)$ & $12 / 19(63.1)$ & $4 / 19(21.1)$ & ns \\
\hline $\begin{array}{l}\text { Photosensitivity } \\
(18 / 51)\end{array}$ & $29 / 66(43.9)$ & $11 / 36$ (30.59 & $7 / 18(38.9)$ & $11 / 18(61.1)$ & $0 / 18(0)$ & ns \\
\hline Oral ulcers (17/51) & $25 / 68(36.7)$ & $15 / 34(44.1)$ & $3 / 17(17.6)$ & 13/17 (76.4) & $1 / 17(6)$ & ns \\
\hline $\begin{array}{l}\text { Chilblain lesions } \\
(6 / 51)\end{array}$ & $35 / 90(8.8)$ & $5 / 12(41.7)$ & $1 / 6(16.7)$ & $5 / 6$ (83.3) & $0 / 6(0)$ & ns \\
\hline Xerostomia (17/51) & $24 / 68(35.3)$ & $16 / 34(47)$ & $3 / 17(17.6)$ & $12 / 17(70.6)$ & $2 / 17(11.8)$ & ns \\
\hline $\begin{array}{l}\text { Xeropthalmia } \\
(11 / 51)\end{array}$ & $29 / 80(36.2)$ & $11 / 22(50)$ & 2/11 (18.2) & $7 / 11$ (63.6) & 2/11 (18.2) & ns \\
\hline $\begin{array}{l}\text { Raynaud's } \\
\text { phenomenon } \\
(15 / 51)\end{array}$ & $27 / 72(37.5)$ & $13 / 30(43.3)$ & $5 / 15(33.3)$ & $7 / 15(46.7)$ & $3 / 15(20)$ & ns \\
\hline Fatigue $(34 / 51)$ & $16 / 34(47)$ & $24 / 68(35.9)$ & 13/34 (38.2) & $18 / 34(52.9)$ & $3 / 34(8.9)$ & ns \\
\hline Serositis $(17 / 51)$ & 29/68 (42.6) & $11 / 34(32.3)$ & $8 / 17(47)$ & $7 / 17(41.2)$ & 2/17 (11.8) & ns \\
\hline $\begin{array}{l}\text { Glomerulonephritis } \\
(18 / 51)\end{array}$ & $27 / 66(41)$ & $13 / 36(36.1)$ & 6/18 (33.3) & $11 / 18(61.1)$ & $1 / 18(5.6)$ & ns \\
\hline NPSLE (8/51) & $28 / 86(32.5)$ & $12 / 16(75)$ & $1 / 8(12.5)$ & $2 / 8(25)$ & $5 / 8(62.5)$ & $0.002^{\star \star}$ \\
\hline Leukopenia (19/51) & 23/64 (35.9) & $17 / 38(44.7)$ & $5 / 19(26.3)$ & $11 / 19(57.9)$ & $3 / 19(15.8)$ & ns \\
\hline $\begin{array}{l}\text { Lymphopenia } \\
(11 / 51)\end{array}$ & $30 / 90(33.3)$ & 10/22 (45.4) & 2/11 (18.2) & $8 / 11(72.7)$ & $1 / 11(9.1)$ & ns \\
\hline $\begin{array}{l}\text { Thrombocytopenia } \\
(8 / 51)\end{array}$ & $32 / 86(37.2)$ & $8 / 16(50)$ & $1 / 8(12.5)$ & $6 / 8(75)$ & $1 / 8(12.5)$ & ns \\
\hline $\begin{array}{l}\text { Hemolytic anemia } \\
(6 / 51)\end{array}$ & $34 / 90(37.7)$ & $6 / 12(50)$ & $1 / 6(16.7)$ & $4 / 6(66.6)$ & $1 / 6(16.7)$ & ns \\
\hline $\begin{array}{l}\text { Positive } \\
\text { anti-dsDNA } \\
\text { positivity (49/51) } \\
\end{array}$ & $3 / 4(75)$ & $37 / 98(37.7)$ & $18 / 49(36.7)$ & $25 / 49(51)$ & $6 / 49(12.3)$ & ns \\
\hline $\begin{array}{l}\text { Positive } \\
\text { antiphospholipid } \\
\text { antibodies }(28 / 51)\end{array}$ & $14 / 46(30.4)$ & $26 / 56(46.4)$ & $6 / 28(21.4)$ & $17 / 28(60.7)$ & $5 / 28(17.9)$ & ns \\
\hline
\end{tabular}

MAF, minor allele frequency; NPSLE, neuropsychiatric systemic lupus erythematosus. *P value was considered significant if $<0.0027$ (adjusted with Bonferroni correction); ${ }^{* *}$ odds ratio=6.42 95\% confidence interval (1.7-26.2).

the NPSLE patients (8 patients) showed that only one had the homozygotic wildtype form of the rs11797, two were in heterozygosity for the mutated form and the majority of them (5 out of 8) had the SNP mutation in homozygosis.

Cerebrovascular events were the most frequent manifestation among our patients, occurring in 4 of them, followed by seizure disorders in 2 patients, multiplex mononeuropathy and psychosis in 1 patient, and a severe mood and anxiety disorder in 1 patient.

\section{DISCUSSION AND CONCLUSIONS}

Recent papers suggest several clinical, genetic and basic science similarities between AGS and SLE. A strong link between these two diseases is related to the role that IFN- $\alpha$ plays in them. In a recent paper (20), the author defined AGS as an interferonopathy due to an error of nucleic acid metabolism, consequently showing a strong similarity with SLE, where IFN- $\alpha$ is known to have a pathogenic role (21). TREX1 is an exo- 
nuclease and its impairment could result in excessive immune activation, triggering in particular the innate immune response and the IFN- $\alpha$ pathway. In this study we analyzed patients affected by SLE and controls mostly belonging to the Italian population. We identified a novel non synonymous mutation, which was not observed in the 150 controls analyzed in this study or in previously published papers. The substitution is located in a highly conserved functional region (one of the three motifs which form the catalytic site) and causes an amino acid change, with asparagine instead of an aspartic acid. Several mutations located in the same region, both missense and synonymous, have been reported in SLE, AGS, and familial chilblain lupus. A missense heterozygous variation pArg128His was described in a patient with neuropsychiatric SLE (15). The authors considered this novel mutation as potentially pathogenic because of its location in the ExoII and also due to the fundamental role of arginine in this position that provides single-strand DNA for the enzyme active site, as it disrupts the double strand.

The substitution found in our patient is considered as damaging by the prediction software. Each amino acid has its own specific size, charge, and hydrophobicity value. The original wild-type residue and the newly introduced mutant residue differ in these properties: the aspartic acid residue was negatively charged, whereas the mutant residue (asparagine) is neutral. The mutation is located in a crucial site for the normal enzyme activity, because it affects one of the four metal ion-binding residues of the TREX1 gene: Asp-18, Glu20, Asp-130 and Asp-200 (22). Mutations of the other three amino acids have been identified in patients with dominant familial chilblain lupus and AGS (7, 13, 23-25). An in vitro functional analysis has been performed for the pAsp200Asn mutation, revealing a strong decrease (200-fold) in the enzyme activity for the mutation also in heterozygosity. It can be speculated that also our newly described mutation is able to affect the physiological role of the enzyme, and that a defective TREXI may result in the impairment of the clearance of ssDNA or dsDNA.

We also confirmed the presence of two synonymous mutations, which were reported by our cohort with a similar rate among patients and controls and showed a frequency similar to that of a large multiancestral cohort (9). Synonymous SNPs do not cause an amino acid change, and the role of these mutations in the pathogenesis of diseases is still debated.

AGS and SLE share many similarities, for instance their neurological phenotype. Seizures represent a diagnostic criterion for SLE and are quite often observed in AGS patients. Moreover, neuroimaging findings in SLE patients include white matter lesions, calcifications and cerebral atrophy, which are commonly observed in patients with AGS. In a large multi-ancestral cohort the authors hypothesized that lupus patients with neuropsychiatric manifestations might be enriched for risk alleles in the TREX1 gene. Indeed, the analysis of common SNPs revealed a relatively common risk haplotype among European lupus

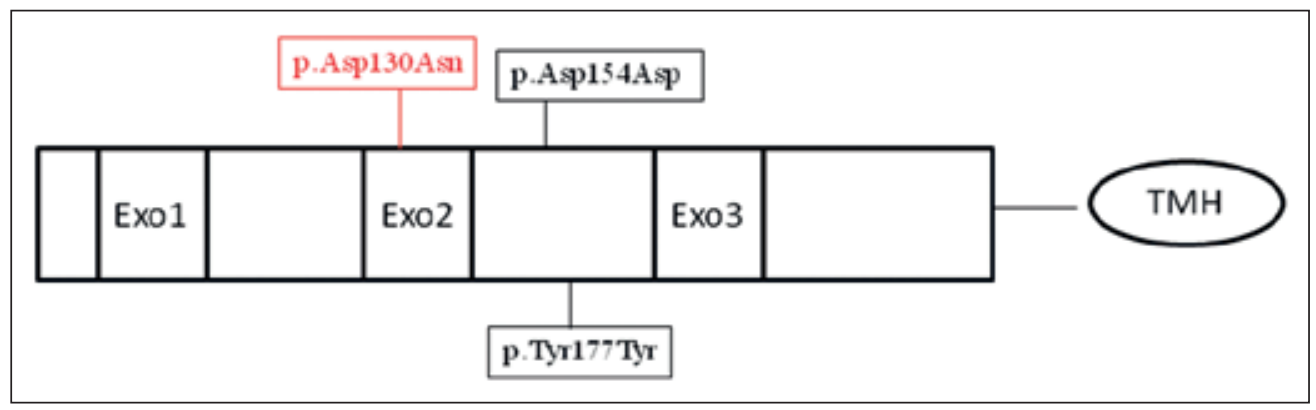

Figure 1 - Scheme of the TREX1 mutations found in this study. Exo1, 2, 3, exonic region; TMH, transmembrane domain. 
patients with seizures (58\% in SLE patients with seizures compared with $45 \%$ in controls). We were not able to replicate these findings in our study for several reasons: firstly the risk haplotype includes SNPs belonging to the TREX1 gene, but also the ATRIP gene, which is closely linked with the exonuclease that was not analyzed in the present study. Secondly we evaluated a relatively small sample of SLE patients, making it difficult to find all the SNPs of the risk haplotype. We reported, however, an association between the mutated common SNP rs11797, a SNP also included in the previously mentioned haplotype, and neurological manifestations. The risk allele was more frequently found among patients with neurological manifestations of SLE than in SLE patients without these manifestations. Moreover, among the 8 SLE patients with NPSLE, only one had the homozygous wild-type form of rs11797.

In conclusion the present study confirms the presence of TREXI mutations in SLE patients (Fig. 1). We reported a potentially pathogenic, novel variation in one of our patients. In our cohort, however, the de novo mutation was found in a patient without a history of neuropsychiatric SLE. We also confirmed the possible role of TREXI gene mutations in a subset of SLE patients with neurological manifestations. Further studies are required to assess the functional relevance of the detected variant and the potential association with SLE.

Conference presentation: L SIR Congress, 2013.

Conflict of interest: the authors declare that there are no conflicts of interest.

\section{REFERENCES}

1. Hoss M, Robins P, Naven TJ, Pappin DJ, Sgouros J, Lindahl T. A human DNA editing enzyme homologous to the Escherichia Coli DnaQ/MutD protein. EMBO J. 1999; 18: 3868-75.

2. Chowdhury D, Beresford PJ, Zhu P, Zhang D, Sug J, Demple B, et al. The exonuclease TREX1 is in the SET complex and acts in concert with NM23-H1 to degrade DNA during granzyme a-mediated cell death. Mol Cell. 2006; 23: 133-42.

3. Stetson DB, Ko JS, Heidmann T, Medzhitov R. Trex1 prevents cell-intrinsic initiation of autoimmunity. Cell. 2008; 134: 587-98.

4. Morita M, Stamp G, Robins P, Dulic A, Rosewell I, Hrivnak G, et al Gene-targeted mice lacking the Trex 1 (DNase III) $3^{\prime} \rightarrow 5^{\prime}$ DNA exonuclease develop inflammatory myocarditis. Mol Cell Biol. 2004; 24: 671927.

5. Crow YJ, Hayward BE, Parmar R, Robins P, Leitch A, Ali M, et al Mutations in the gene encoding the 3'-5' DNA exonuclease TREX1 cause Aicardi-Goutières syndrome at the AGS1 locus. Nat Genet. 2006; 38: 917-20.

6. Rice G, Patrick T, Parmar R, Taylor CF, Aeby A, Aicardi J, et al Clinical and molecular phenotype of Aicardi-Goutières syndrome. Am J Hum Genet. 2007; 81: 713-25.

7. Rice G, Newman WG, Dean J, Patrick T, Parmar R, Flintoff K, et al. Heterozygous mutations in TREX1 cause familial chilblain lupus and dominant Aicardi-Goutieres syndrome. Am J Hum Genet. 2007; 80: 811-5.

8. Lee-Kirsch MA, Gong M, Chowdhury D, Senenko L, Engel K, Lee YA, et al Mutations in the gene encoding the 3'-5' DNA exonuclease TREX1 are associated with systemic lupus erythematosus. Nat Genet. 2007; 39: 1065-7.

9. Namjou B, Kothari PH, Kelly JA, Glenn SB, Ojwang JO, Adler A, et al. Evaluation of the TREX1 gene in a large multi-ancestral lupus cohort. Genes Immun. 2011; 12: 270-9.

10. Barizzone N, Monti S, Mellone S, Godi M, Marchini M, Scorza R, et al. Rare variants in the TREX1 gene and susceptibility to autoimmune diseases. Biomed Res Int. 2013; 2013: 471703.

11. Aicardi J, Goutières F. A progressive familial encephalopathy with calcifications of the basal ganglia and chronic cerebrospinal fluid lymphocytosis. Ann Neurol. 1984; 15: 49-54.

12. Rice GI, del Toro Duany Y, Jenkinson EM, Forte GM, Anderson BH, Ariaudo G, et al. Gain-of-function mutations in IFIH1 cause a spectrum of human disease phenotypes associated with upregulated type I interferon signaling. Nat Genet. 2014; 46: 503-9.

13. Ramantani G, Kohlhase J, Hertzberg C, Innes AM, Engel K, Hunger S, et al. Expanding the phenotypic spectrum of lupus erythematosus in Aicardi-Goutières syndrome. Arthritis Rheum. 2010; 62: 1469-77.

14. De Laet C, Goyens P, Christophe C, Ferster A, Mascart F, Dan B. Phenotypic overlap between infantile systemic lupus erythematosus and Aicardi-Goutieres syndrome. Neuropediatrics 2005; 36: 399-402.

15. de Vries B, Steup-Beekman GM, Haan J, Bollen EL, Luyendijk J, Frants RR, et al. TREX1 
gene variant in neuropsychiatric systemic lupus erythematosus. Ann Rheum Dis. 2010; 69: 1886-7.

16. Hochberg MC. Updating the American College of Rheumatology revised criteria for the classification of systemic lupus erythematosus. Arthritis Rheum. 1997; 40: 1725.

17. Petri M, Orbai AM, Alarcón GS, Gordon C, Merrill JT, Fortinet PR, et al. Derivation and validation of the Systemic Lupus International Collaborating Clinics classification criteria for systemic lupus erythematosus. Arthritis Rheum. 2012; 64: 2677-86.

18. Rost B, Yachdav G, Liu J. The predict protein server. Nucleic Acids Res. 2004; 32: W321-6.

19. Venselaar H, Te Beek TA, Kuipers RK, Hekkelman ML, Vriend G. Protein structure analysis of mutations causing inheritable diseases. An e-Science approach with life scientist friendly interfaces. BMC Bioinformatics. 2010;11: 548 .

20. Crow YJ. Type I interferonopathies: a novel set of inborn errors of immunity. Ann N Y Acad Sci. 2011; 1238: 91-8.

21. Banchereau J, Pascual V. Type I interferon in systemic lupus erythematosus and other autoimmune diseases. Immunity. 2006; 25: 38392.

22. Orebaugh CD, Fye JM, Harvey S, Hollis T, Perrino FW. The TREX1 exonuclease R114H mutation in Aicardi-Goutières syndrome and lupus reveals dimeric structure requirements for DNA degradation activity. J Biol Chem. 2011; 286: 40246-54.

23. Lee-Kirsch MA, Chowdhury D, Harvey S, Gong M, Senenko L, Engel K, et al. A mutation in TREX1 that impairs susceptibility to granzyme A-mediated cell death underlies familial chilblain lupus. J Mol Med (Berl). 2007; 85: 531-7.

24. Günther C, Meurer M, Stein A, Viehweg A, Lee-Kirsch MA. Familial chilblain lupus - a monogenic form of cutaneous lupus erythematosus due to a heterozygous mutation in TREX1. Dermatology. 2009; 219: 162-6.

25. Haaxma CA, Crow YJ, van Steensel MA, Lammens MM, Rice GI, Verbeek MM, et al. A de novo p.Asp18Asn mutation in TREX1 in a patient with Aicardi-Goutières syndrome. Am J Med Genet. 2010; 152A: 2612-7. 